\title{
Breast metastasis from signet ring cell carcinoma of the urinary bladder: A case report
}

\author{
YAOMIN CHEN, HAIYAN WEI, JUN LI, XIAOJIAO LIU and PEIFEN FU \\ Department of Breast Surgery, The First Affiliated Hospital, College of Medicine, \\ Zhejiang University, Hangzhou, Zhejiang 310003, P.R. China
}

Received October 1, 2015; Accepted January 13, 2016

DOI: $10.3892 / \operatorname{mco} .2016 .884$

\begin{abstract}
Carcinoma of the bladder metastatic to the breast is only sporadically reported in the literature. To the best of our knowledge, the present report is the first described case of signet ring cell carcinoma of the urinary bladder metastasizing to the breast. The patient was a 43-year-old woman who underwent transurethral partial cystectomy for signet ring cell carcinoma of the urinary bladder and adjuvant chemotherapy with cisplatin and gemcitabine. At 7 months postcystectomy, the patient presented with a solitary nodule in the right breast. Following transdermic core needle puncture biopsy of the lesion and histological examination, the tumor was found to be composed of signet ring cells, which were similar to the cells in the original cystectomy specimen.The patient underwent mastectomy without further chemotherapy and has remained free from metastasis to other organs during 1 year follow-up.
\end{abstract}

\section{Introduction}

Breast cancer is the most common malignancy in women, whereas metastases to the breast from extramammary malignancies are very rare, accounting for only $0.43 \%$ of all malignant breast tumors (1). The most common primary tumors metastasizing to the breast are melanoma (29.8\%), lung carcinoma (16.4\%), gynecological carcinoma (12.7\%), intestinal carcinoma (9.9\%), leukemia and lymphoma (8.4\%), rhabdomyosarcoma (7.3\%) and renal cell carcinoma (1.5\%). Clinically, the lesions are usually round, painless, without skin retraction or associated adenopathies (33\%). On imaging, metastatic lesions are frequently single. Diagnosis, sometimes suggested by morphological study of the tumor, is confirmed by immunohistochemistry. Systemic metastases are common

Correspondence to: Professor Peifen Fu, Department of Breast Surgery, The First Affiliated Hospital, College of Medicine, Zhejiang University, 79 Qingchun Road, Hangzhou, Zhejiang 310003, P.R. China

E-mail: vipdoctor@163.com

Key words: breast metastasis, signet ring cell carcinoma of the urinary bladder and they are associated with a shorter survival (1). Breast metastasis originating from urinary bladder carcinoma has previously been described (2-4). We herein report the case of a 43 year-old woman with signet ring cell carcinoma of the urinary bladder metastasizing to the breast.

\section{Case report}

A 43 year-old female patient was referred to our hospital in February, 2014 with a 2-month history of intermittent painless total gross hematuria, frequency and urgency. Ultrasonography and a computed tomography scan revealed a solitary mass located below the urachus, without lymph node enlargement or distant metastases. A histopathological specimen obtained by cystoscopic examination was diagnosed as a poorly differentiated mucin-secreting adenocarcinoma of the signet ring cell type. A complete gastrointestinal endoscopic evaluation and analysis of tumor markers were performed to exclude an extravesical primary tumor, but no other primary site was identified. The tumor was therefore treated as a primary signet ring cell carcinoma of the urinary bladder and the patient underwent transurethral partial cystectomy. The histopathological examination of the surgical specimen revealed an adenocarcinoma composed of signet ring cells, with an abundant mucin pool, invading the deep muscular layer (Fig. 1). Adjuvant chemotherapy was then performed, with four cycles of cisplatin $\left(100 \mathrm{mg} / \mathrm{m}^{2}\right.$ on day 1$)$ and gemcitabine $\left(1,000 \mathrm{mg} / \mathrm{m}^{2}\right.$ on days 1 and 8), every 21 days. In September, 2014 a solitary subcutaneous mass $(1.9 \times 1.0 \mathrm{~cm})$ was palpated in the right breast. As it is difficult to differentiate metastatic lesions in the breast from primary breast cancer or benign lesions by ultrasonic appearance (Fig. 2) and physical examination, a transdermic core needle puncture biopsy of the breast lump was performed, which revealed the presence of a signet ring cell carcinoma metastatic from the bladder (Fig. 3). The patient underwent mastectomy. No further chemotherapy was performed and the patient remained free from metastasis to other organs during 1 year follow-up.

\section{Discussion}

Signet ring cell carcinoma is a relatively rare neoplasm that rarely occurs in the urinary bladder, comprising only $0.24-2 \%$ of all primary epithelial urinary bladder tumors $(5,6)$, with 


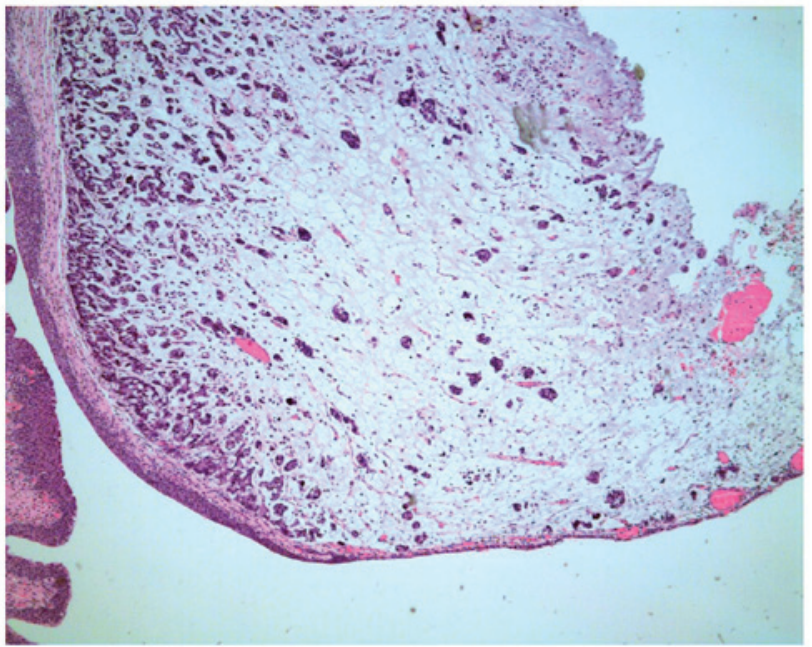

Figure 1. Poorly differentiated mucin-secreting adenocarcinoma of the signet ring cell type $\left(\mathrm{CK}_{20}{ }^{+}, \mathrm{CDX}_{2}{ }^{+}, \mathrm{CEA}^{+}, \mathrm{P}^{-}, \mathrm{CK}^{+}\right)$. Staining, hematoxylin and eosin; original magnification, $\mathrm{x} 50 . \mathrm{CK}$, cytokeratin; CDX2 caudal type homeobox 2; CEA, carcinoembryonic antigen.

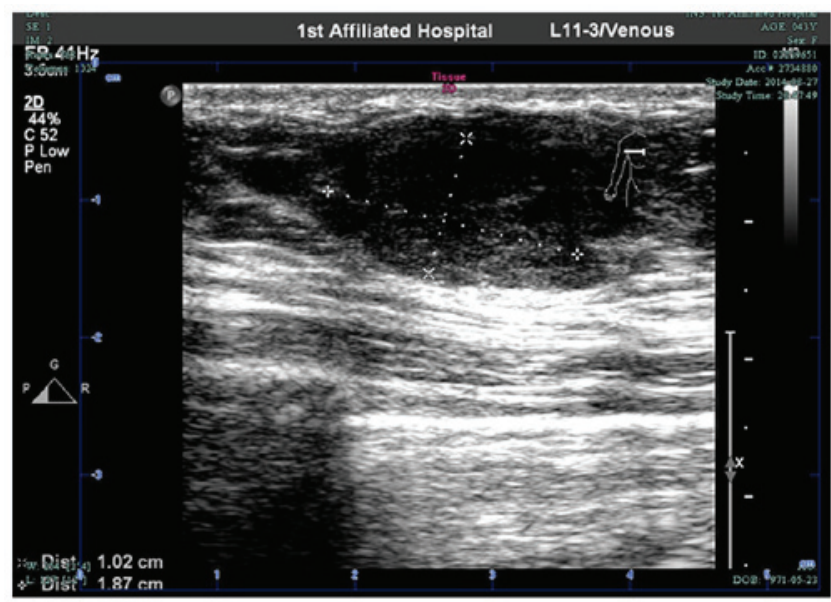

Figure 2. Ultrasonogram showing a lesion located in the upper right breast.

$<100$ cases reported in the literature since the first 2 cases reported by Saphir in 1955 (7). The most frequent sites of metastasis from primary bladder carcinoma are the lymph nodes, liver, lung and bone (8). This type of neoplasm generally occurs in middle age, is usually diagnosed at an advanced stage and is usually associated with a poor prognosis (9). The first case of carcinoma of the bladder metastatic to the male breast was reported by Truesdale et al in 1979 (2). At 7 months postcystectomy, a poorly differentiated transitional cell carcinoma metastatic to the breast and suprasternal area was detected. The first detailed description of a case of transitional cell carcinoma of the bladder metastatic to the female breast was reported by Erkman et al in 1982 (3). The patient was a 43-year-old woman who underwent radical cystectomy 3 months prior to presentation with two asymptomatic right breast masses as the first evidence of widely metastatic bladder carcinoma. Recently, breast metastasis originating from urinary bladder carcinoma has been described sporadically (10). To the best of our knowledge, the present case represents the first description of a primary signet ring cell

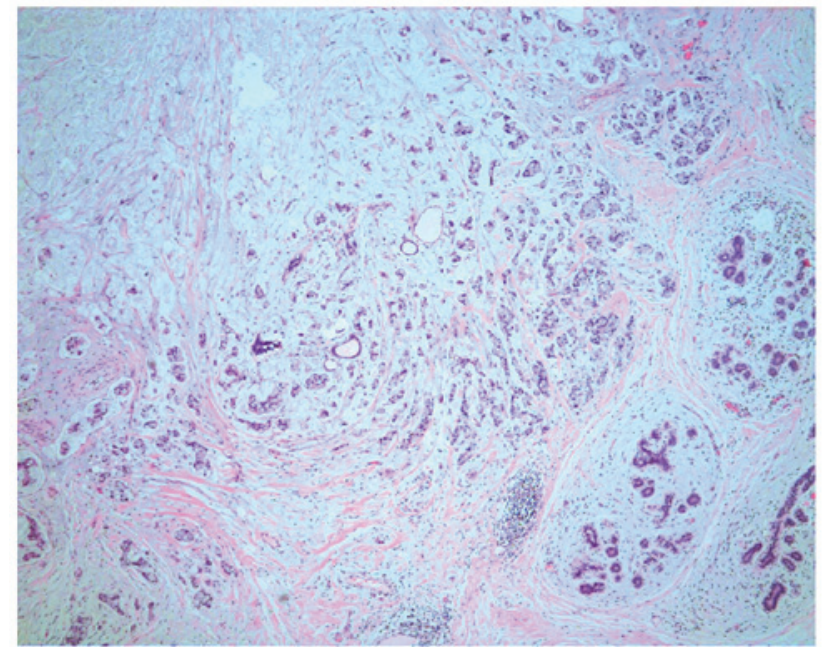

Figure 3. Metastatic adenocarcinoma composed of signet ring cells with an abundant mucin pool (ER', PR', CK5 ${ }^{-}$, E-cadherin ${ }^{+}, \mathrm{G}^{-} 5^{-}, \mathrm{P}^{-} 20^{-}, \mathrm{CDX}^{+}$, $\left.\mathrm{MMG}^{-}\right)$. Staining, hematoxylin and eosin;original magnification, $\mathrm{x} 50$. ER, estrogen receptor; PR, progesterone receptor; $\mathrm{CK}$, cytokeratin; CDX2 caudal type homeobox 2; MMG, mammaglobin.

carcinoma of the urinary bladder with breast metastases. In addition, the patient's presentation with a solitary metastatic breast nodule without widespread dissemination is inconsistent with the natural history of metastatic bladder carcinoma, although 1 year follow-up period was relatively shorter. The ultrasonic appearance of breast metastasis exhibits no typical characteristics. Therefore, the differentiation of metastatic lesions in the breast from primary breast cancer or other types of cancer depends on histopathological examination.

\section{References}

1. Koch A, Richter-Marot A, Wissler MP, Baratte A and Mathelin C: Mammary metastasis of extramammary cancers: current knowledge and diagnostic difficulties. Gynecol Obstet Fertil 41: 653-659, 2013.

2. Truesdale BH, Johnson RD, Evins SC, et al: Carcinoma of bladder metastatic to breast. Urology 13: 430-431, 1979.

3. Erkman AW, Markman M, Murphy JB and Lenhard RE Jr: Transitional cell carcinoma of the bladder metastatic to the breast. Med Pediatr Oncol 10: 141-144, 1982.

4. Domínguez Anguiano M, Campoy Martínez P, Cañizares Diaz I, Ramírez Mendoza A and Soltero González A: Breast metastasis of bladder carcinoma: Report of a case. Arch Esp Urol 52: 985-987, 1999.

5. Sunghwan J, Soojin J, Kweonsik M, Jae-il C, Sunghyup C and Dongil K: Primary signet ring cell carcinoma of the urinary bladder. Korean J Urol 50: 188-191, 2009.

6. Torenbeek R, Koot RA, Blonjous CE, De Bruin PC, Newling DW and Meijer CJ: Primary signet-ring cell of the urinary bladder. Histopathology 28: 33-40, 1996.

7. Saphir O: Signet-ring cell carcinoma of the urinary bladder. Am J Pathol 31: 223-231, 1955.

8. Babian RJ, Johnson DE, Llamas L and Ayala AG: Metastases from transitional cell carcinoma of urinary bladder. Urology 16: 142-144, 1980.

9. El-Mekresh MM, El-Baz MA, Abol-Enein H and Ghoneim MA: Primary adenocarcinoma of the urinary bladder: A report of 185 cases. British J Urol 82: 206-212, 1998.

10. Cappabianca S, Grassi R, D'Alessandro P, Del Vecchio A, Maioli A and Donofrio V: Metastasis to the male breast from carcinoma of the urinary bladder. Br J Radiol 73: 1326-1328, 2000. 